\title{
Antimicrobial Resistance and Presence of Class 1 Integrons in Salmonella Serovars Isolated from Clinical Cases of Animals and Humans in North Dakota and Uganda
}

\author{
Mahero $\mathbf{M}^{1}$, Byarugaba $\mathrm{DK}^{2}$, Doetkott $\mathrm{DK}^{3}$, Olet $\mathrm{S}^{3}$ and Khaitsa $\mathrm{ML}^{4 *}$ \\ ${ }^{1}$ The Great Plains Institute of Food Safety, North Dakota State University, USA \\ ${ }^{2}$ Department of Veterinary Parasitology and Microbiology, Makerere University, Uganda. \\ ${ }^{3}$ Department of Statistics, North Dakota State University, USA \\ ${ }^{4}$ Department of Veterinary and Microbiological Sciences, North Dakota State University, USA
}

\begin{abstract}
Back ground: Salmonellae are one of the leading causes of food borne illness worldwide and have been used as indicator organisms for studying antimicrobial resistance (AMR) trends. In the United States, Salmonella are among organisms currently under public health surveillance for AMR.

Objectives: The objective of this study was to characterise AMR patterns of Salmonella isolates from animals and humans in North Dakota (ND), and Kampala, Uganda and determine the association between the observed AMR and presence of class 1 and 2 integrons.

Methods: Salmonella isolates were collected from the Veterinary Diagnostic Laboratory (VDL) at North Dakota State University and the North Dakota Department of Health, from 2003 to2008. Additional samples were also retrieved from archives at the Microbiology Department, Faculty of Veterinary Medicine at Makerere University in Kampala, Uganda. AMR profiles were determined using a panel of 15 antimicrobials. Screening for the class 1 and 2 integrons was done using PCR with primers specific for the int1 and int2.

Results: Out of 359 Salmonella isolates tested $36.2 \%$ were resistant to at least 2 antimicrobials. The highest resistance frequency was seen against Tetracycline (39.6\%) and Streptomycin (34.7\%). A total of 20.7\% (57/276) of the ND samples tested positive for presence of class 1 integrons and was significantly associated $(p<0.05)$ with AMR to Ampicillin, Kanamycin, Tetracycline and Sulfisoxazole. Of all Ugandan Salmonella isolates tested $(94.4 \%$ $68 / 72$ ) were resistant to $\geq 2$ antimicrobials with highest resistance observed against Sulfisoxazole and TrimethoprimSulphamethoxazole. Presence of class 1 integron was significantly associated $(p<0.05)$ with AMR to Tetracycline and Amoxicillin. DNA sequencing of the class 1 integron variable regions identified several resistance genes including aadA1, dfrA7, and dfrA5 genes. Conclusion: These results signal serious implications for treatment of salmonellosis in both public and animal health.
\end{abstract}

Keywords: Antimicrobial resistance; Salmonella; Integron 1 North Dakota; Uganda

\section{Introduction}

Antimicrobial resistance (AMR) is a natural consequence of infectious agents' adaptation to exposure to antimicrobials used in medicine, food animals, food processing, crop production and the environment [1-4]. There has been a decline in effectiveness of existing antimicrobial agents and thus infections have become more difficult and expensive to treat and epidemics have become harder to control $[5,6$, 7]. As a result, in 1996, the United States (US) established The National Antimicrobial Resistance Monitoring System (NARMS), a national public health surveillance system that tracks antibiotic resistance in food borne bacteria [8]. The NARMS program is a partnership between the US Food and Drug Administration (FDA), the Centers for Disease Control and Prevention (CDC), and the US Department of Agriculture (USDA) that monitors antimicrobial susceptibility among enteric bacteria from humans, retail meats, and food animals [8]. NARMS also collaborates with AMR monitoring systems in other countries, to work towards international harmonization of testing and reporting [8]. Salmonella are among the major bacteria currently under surveillance. Salmonella are among organisms currently under public health surveillance for antimicrobial resistance [8].

Salmonella has been reported as one of the leading causes of food borne illness in the US [9] and worldwide $[1,6,10]$. In the United States of America (US), the major pathogens that have been associated with food borne outbreaks are comprised of viruses, bacteria, parasites, toxins, metals and prions [9]. Of these 7 major food pathogens (Campylobacter jejuni, Clostridium pefrigens, Escherichia coli, Listeria monocytogenes, Salmonella, Staphlococcus aureus and Toxoplasma gondii) are known to cause 3.3-12.3 million cases of food borne illness and up to 3900 deaths, with an estimated total cost of \$6.5-\$ 34.9 billion (1995 US\$) annually [9]. Salmonella is responsible for approximately 1.4 million illnesses, 17,000 hospitalisations and 590 deaths in the US annually [9]. According to Food Net (Food borne Diseases Active Surveillance Network), Salmonella prevalence has consistently remained high in comparison to the other food borne pathogens despite various intervention measures [2]. In 2011 estimates,

*Corresponding author: Margaret L. Khaitsa, Department of Veterinary and Microbiological Sciences, North Dakota State University, USA, Tel: (701) 231-5946; Fax (701) 231-9692; E-mail: Margaret.Khaitsa@ndsu.edu

Received August 05, 2013; Accepted August 19, 2013; Published August 21 2013

Citation: Mahero M, Byarugaba DK, Doetkott DK, Olet S, Khaitsa ML (2013) Antimicrobial Resistance and Presence of Class 1 Integrons in Salmonella Serovars Isolated from Clinical Cases of Animals and Humans in North Dakota and Uganda. Clin Microbial 2: 128. doi: 10.4172/2327-5073.1000128

Copyright: ( 2013 Mahero M, et al. This is an open-access article distributed unde the terms of the Creative Commons Attribution License, which permits unrestricted use, distribution, and reproduction in any medium, provided the original author and source are credited. 
Citation: Mahero M, Byarugaba DK, Doetkott DK, Olet S, Khaitsa ML (2013) Antimicrobial Resistance and Presence of Class 1 Integrons in Salmonella Serovars Isolated from Clinical Cases of Animals and Humans in North Dakota and Uganda. Clin Microbial 2: 128. doi: 10.4172/23275073.1000128

Page 2 of 7

the leading causes of hospitalization were nontyphoidal Salmonella spp. (35\%), and the leading causes of death were nontyphoidal Salmonella spp. (28\%) [11]. Unfortunately the burden of disease, associated mortality and epidemiology in sub-Saharan Africa is unknown although outbreaks with high case fatality rates are reported to the World Health Organisation [4].

Previous studies have demonstrated the presence of AMR in Salmonella and other bacteria of family Enterobacteriaceace. Often, this resistance is encoded by integrons that occur on plasmids or that are integrated into the bacterial chromosome [12]. Although this AMR genetic mechanism has repeatedly been demonstrated $[13,14]$ few epidemiological studies [15] have been conducted to quantify the phenotypic resistance that is attributed to these genetic structures. Additionally, there are other genetic mechanisms that contribute to the observed resistance [13] which also need to be characterized. Moreover, few studies have been done in sub Saharan Africa to investigate the role of integrons in AMR acquisition by food borne pathogens.

The objective of this study was to characterize Salmonella isolates from the US (North Dakota) and Uganda (Kampala) based on AMR, presence of integrons and genetic sequencing of the integron gene cassettes.

\section{Materials and Methods}

\section{Study design}

This was a retrospective case series. Salmonella isolates included in the study were collected either as part of diagnostic procedures for large animal patients or as part of an active hospital surveillance program, and were obtained from the Veterinary Diagnostic Laboratory (VDL) at NDSU (North Dakota State University) and the North Dakota Department of Health (NDDoH) respectively. These isolates had been previously obtained from clinical cases of bovine and human salmonellosis that were presented at the VDL and $\mathrm{NDDoH}$ from 2003-2008. All isolates had been cultured and characterised according to methods optimised for Salmonella detection $[5,13]$. Additionally, archived samples from the Department of Microbiology, at the Faculty of Veterinary Medicine, Makerere University in Kampala Uganda were used. Uganda was chosen as a typical example of a developing nation and also due to the preexisting partnership between the research team at NDSU Department of Veterinary Microbiological Sciences (VMS) and Makerere University Kampala Uganda. Approval to carry out this project was obtained from the NDSU Institutional Review Board and the Uganda National Council of Science and Technology.

\section{Antimicrobial susceptibility testing}

Antimicrobial resistance of each Salmonella isolate was determined using a panel of 15 antimicrobials (Sensitire, Trek Diagnostics System, Westlake, Ohio). Each CMV1AGMF plate used for resistance screening contained a full range of minimum inhibitory concentrations (MIC). The panel consisted of 96-well microtitre plate containing different antimicrobials over a wide range of concentrations. The inoculation of the panels was done in accordance with the manufacturer's instructions (Trek Diagnostics). The antimicrobials tested were Amikacin, Amoxicillin/clavulanic acid, Ampicillin, Ceftiofur, Ceftriaxone, Chloramphenicol, Ciprofloxacin, Gentamicin, Kanamycin, Nalidixic acid, Streptomycin, Sulfizoxazole, Tetracycline, and Trimethoprim / sulfamethoxizole. Antimicrobial resistance was interpreted using Clinical and Laboratory Standards Institute (CLSI, formerly NCCLS) standards. For antimicrobial agents without CLSI approved standards, NARMS interpretive criteria were used.

\section{Class 1 and 2 integron detection}

Class 1 and 2 integron detection was accomplished by using PCR primers specific for class 1 and 2 intergrase; 280 -bp and $233 \mathrm{bp}$ amplicon respectively. The protocol used was previously described by Miko et al [16]. Screening for the class 1 and 2 integrons was carried out using PCR with primers specific for the intland 2 [14]. Briefly, in order to extract the DNA, Proteinase $\mathrm{K}$ was added to the samples and heated at $94^{\circ} \mathrm{C}$ for $5 \mathrm{~min}$. Thereafter amplifications were performed in $23 \mu \mathrm{L} 5 \mathrm{X}$ of Taq PCR (Polymerase Chain Reaction) Master Mix (Promega), 10 pmol/L each primer, and $2 \mu \mathrm{l}$ template DNA followed by 35 cycles of $1 \mathrm{~min}$ at $94^{\circ} \mathrm{C}, 1 \mathrm{~min}$ at $55^{\circ} \mathrm{C}$, and $30 \mathrm{~s}$ at $72^{\circ} \mathrm{C}$. PCR products were analyzed by gel electrophoresis with $1.5 \%$ agarose gels. All PCRs included both positive and negative controls.

\section{DNA purification and sequencing}

A representative sample of 24 isolates of Salmonella was selected according to the size (gene profile) of the gene within each isolate and the host from which the isolate was obtained; The single reaction PCR was followed to amplify the Conserved Sequence as previously described by Nde et al., [17]. The amplification products were purified using The Wizard ${ }^{\star}$ SV Gel and PCR Clean-Up System according to manufacturer's instruction. Purified DNA was sent to Macrogen USA for sequencing. The sequences were compared with the data in the Gen Bank (http://www.ncbi.nlm.nih.gov/BLAST).

\section{Data analysis}

Phenotypic resistance was presented as the percentage of the total isolates tested that were resistant. Descriptive statistics of class 1 integrons detected within Salmonella serotypes were computed using Epi Info version 3.3.2 software (Epi Info TM, U.S Center for Disease Control and Prevention (CDC), Atlanta, GA). The association between the observed resistance and the presence and location of Class 1 integrons in the serovars was determined using Chi-square test of independence as previously described by Khaitsa et al [18]. AMR was coded as absent (0) or present (1) with both the resistant and intermediate isolates considered as resistant. Measures of association computed included Attributable fractions and Odds Ratios.

\section{Results}

\section{Antimicrobial susceptibility testing}

Overall, Salmonella isolates from North Dakota exhibited the highest antimicrobial resistance towards Tetracycline (39.60\%),

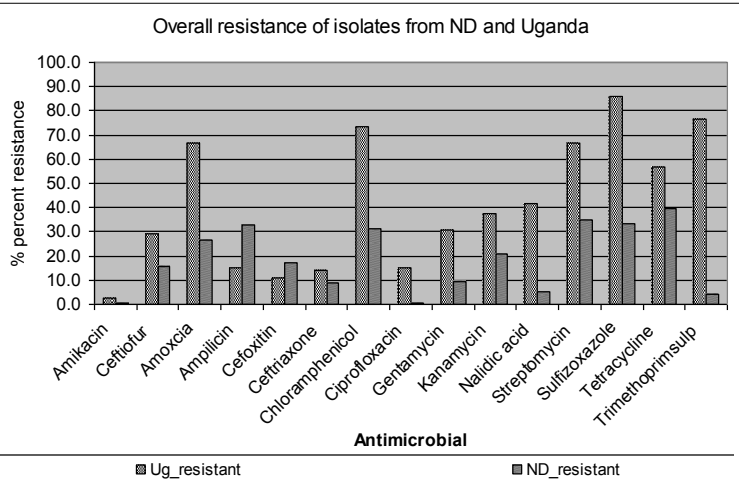

Figure 1: Overall Resistance Profile of Salmonella isolates from North Dakota and Uganda tested against National Antimicrobial Resistance Monitoring Systems panel of antimicrobials. 
Citation: Mahero M, Byarugaba DK, Doetkott DK, Olet S, Khaitsa ML (2013) Antimicrobial Resistance and Presence of Class 1 Integrons in Salmonella Serovars Isolated from Clinical Cases of Animals and Humans in North Dakota and Uganda. Clin Microbial 2: 128. doi: 10.4172/23275073.1000128

Streptomycin (34.70 \%), Sulfisoxazole (33.10\%), Ampicillin (32.60\%) and Chloramphenicol (31.40\%) (Figure1). This pattern was similar to that observed in Salmonella isolates from cattle with the highest resistance observed against Tetracycline (61.0\%, 102/170), Streptomycin $(54.80 \%, 94 / 171)$ (Table1). Among Salmonella isolates from humans, high antimicrobial resistance (AMR) frequencies were reported against Tetracycline 19.40\%, 37/186), Chloramphenicol $(16.70 \%, 31 / 186)$ (Table 1). A substantial proportion of the tested Salmonella isolates showed resistance to several antimicrobials within the critically important agents as follows: Streptomycin $34.70 \%$

\begin{tabular}{|l|c|c|c|c|}
\hline Antimicrobial & \multicolumn{2}{|c|}{ North Dakota (\% Resistant) } & \multicolumn{2}{|c|}{ Uganda (\%Resistant ) N=72 } \\
\hline & $\begin{array}{c}\text { Humans } \\
(\mathrm{N}=186)\end{array}$ & $\begin{array}{c}\text { Cattle } \\
(\mathrm{N}=173)\end{array}$ & $\begin{array}{c}\text { Humans } \\
(\mathrm{N}=58)\end{array}$ & $\begin{array}{c}\text { Cattle } \\
(\mathrm{N}=14)\end{array}$ \\
\hline Amikacin & 0 & 0.58 & 0.0 & 16.7 \\
\hline Ceftiofur & 4.30 & 28.07 & 28.6 & 41.7 \\
\hline Amoxcia & 11.29 & 42.77 & 73.7 & 50.0 \\
\hline Ampicillin & 13.44 & 53.18 & 16.1 & 23.7 \\
\hline Cefoxitin & 5.92 & 29.07 & 8.8 & 25.0 \\
\hline Ceftriaxone & 3.23 & 17.34 & 14.8 & 16.7 \\
\hline Chloramphenicol & 16.67 & 47.37 & 81.0 & 50.0 \\
\hline Ciprofloxacin & 0 & 1.16 & 14.3 & 27.3 \\
\hline Gentamycin & 4.83 & 13.8 & 44.2 & 25.0 \\
\hline Kanamycin & 10.21 & 32.37 & 49.0 & 16.7 \\
\hline Nalidixic acid & 5.38 & 4.62 & 44.9 & 72.7 \\
\hline Streptomycin & 16.37 & 54.83 & 77.2 & 36.4 \\
\hline Sulfizoxazole & 16.13 & 51.45 & 92.9 & 83.3 \\
\hline Tetracycline & 19.35 & 61.27 & 65.4 & 58.3 \\
\hline Trimethoprimsulp & 1.08 & 7.51 & 85.7 & 63.6 \\
\hline
\end{tabular}

Table 1: Overall Resistance Profile of Salmonella isolates from North Dakota and Uganda tested against National Antimicrobial Resistance Monitoring Systems panel of antimicrobials.
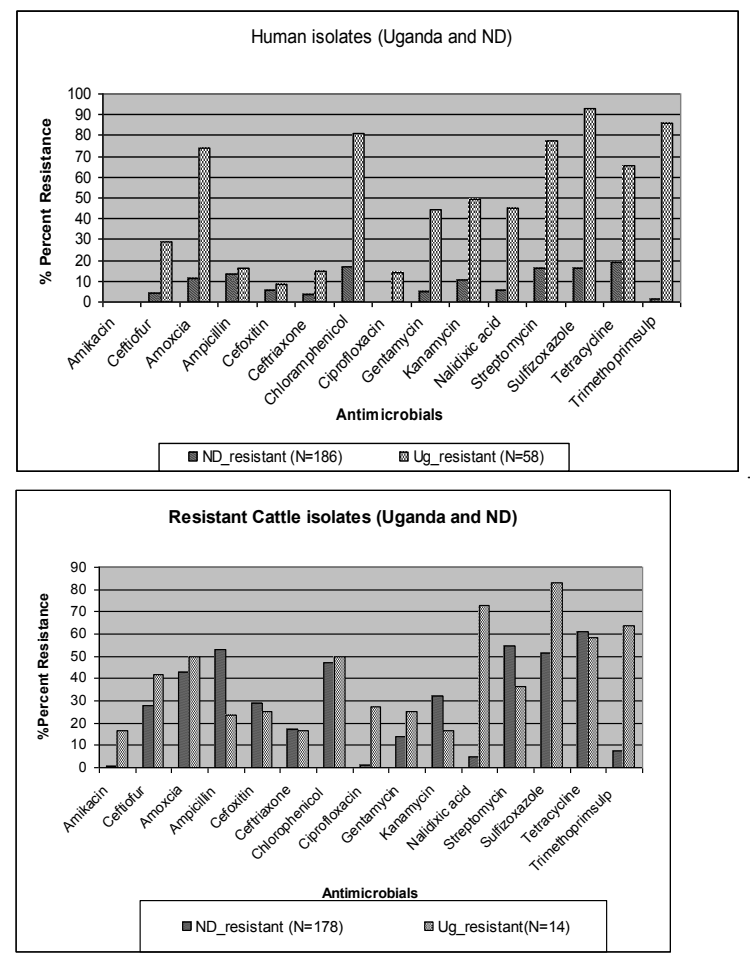

Figure 2: Comparison of Antimicrobial Resistance profiles from cattle and human Salmonella isolates from North Dakota and Uganda.

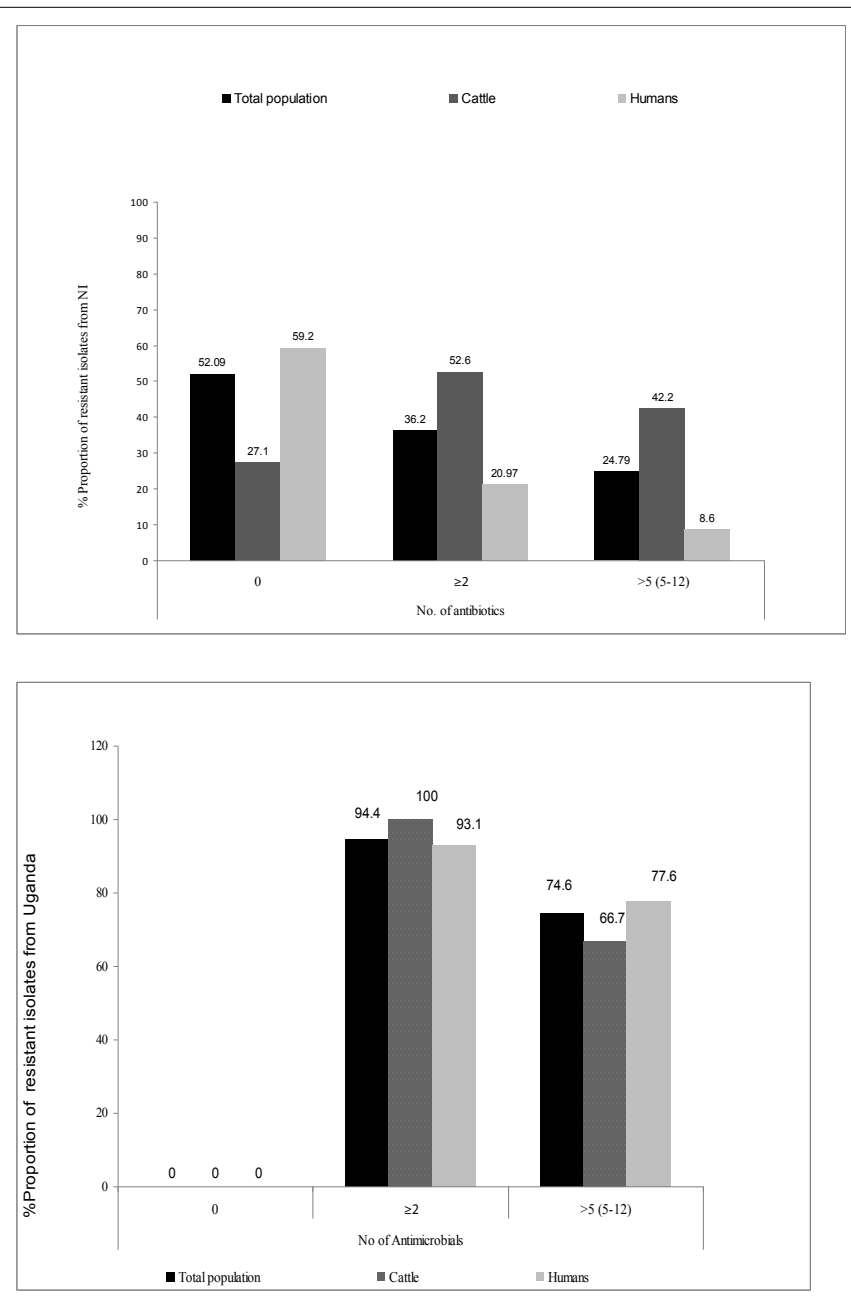

Figure 3: Multidrug Resistance in Salmonella Isolates from North Dakota and Uganda.

(113/326); [54.8\% Cattle 85/155 and 16.4\% Human 28/171], Ampicillin 31.90\% (114/357); [ 52\% Cattle 89/171 and 13.4\% Humans 25/186], Amoxicillin-Clavulanic acid 25.80\% ( 92/357) ; [ 41.5\% Cattle 71/171 and $11.3 \%$ Humans 21/186], Ceftiofur 15.70\% (56/357); [ $28.10 \%$ Cattle 48/171 and 4.30\% Humans 8/186]; Ceftriaxone $9.50 \%(34 / 357)$ [16.4\% Cattle 28/171 and 3.20\% Humans 6/186] (Figure 2). Among the clinically important antimicrobial subclasses- quinolones (represented by Nalidixic acid) and third-generation cephalosporins (represented by Ceftiofur), 10 (5.40\%) of the humans and 7 (4.60\%) of the cattle isolates were resistant to Nalidixic acid, a drug in the same class with Ciprofloxacin. Of the 17 Nalidixic acid resistant isolates, 7 (2.4\%) also had reduced susceptibilities against ciprofloxacin (MIC >0.25) (2.4\%). Also, $56(15.70 \%)$ of all isolates tested were resistant against Ceftiofur, 48 (28.10\%) from cattle and 8 (4.30\%) from humans (Figure 2).

From Uganda high resistance was seen against Sulfisoxazole (86.10\%), Trimethoprim (76.40\%), Chloramphenicol (73.60\%), Streptomycin (66.70\%), Ampicillin (66.70\%) and Tetracycline (56.90\%) (Figure1 and 2). Some of these drugs fall under the WHO described group of critically important drugs in human medicine. The highest resistance was observed against Sulfisoxazole in cattle $(83.3 \%, 8 / 12)$ and humans $(91.2 \%, 52 / 57)$ followed by Trimethoprim in humans $(85.7 \%$, $48 / 12$ ) and Nalidixic acid $(72.73 \%, 8 / 11)$ in cattle (and 4). Relatively high resistance to Ciprofloxacin (a drug of choice for treatment of 
Citation: Mahero M, Byarugaba DK, Doetkott DK, Olet S, Khaitsa ML (2013) Antimicrobial Resistance and Presence of Class 1 Integrons in Salmonella Serovars Isolated from Clinical Cases of Animals and Humans in North Dakota and Uganda. Clin Microbial 2: 128. doi: 10.4172/23275073.1000128

Page 4 of 7

salmonellosis in humans) was seen in cattle-27.30\% (3/11) and $14.29 \%$ (8/576) human isolates. The lowest resistance was recorded against Amikacin and Ceftriaxone $16.70 \%(2 / 12)$ among cattle isolates and Amikacin 0\% (0/56) among human isolates (Table 1).

\section{Multi drug resistance (MDR)}

Of 359 Salmonella isolates from ND tested, $24.79 \%$ (89/359) were resistant to $\geq 5$ antimicrobials while $36.20 \%$ (130/359) were resistant to at least 2 (Figure 3). For cattle and human isolates $52.60 \%(91 / 173)$ and $20.97 \%$ (39/186), respectively, had resistance to $\geq 2$ antimicrobials while $42.20 \%(73 / 173)$ and $8.60 \%(16 / 186)$, respectively, were resistant to $\geq 5$ antimicrobials (Figure 3). Pan susceptible isolates were 27.1\% (66/173) in cattle and 59.2\% (121/186) in humans (Figure 3). The most common MDR phenotype among the Salmonella isolates was the classic ACSSuT (Ampicillin, Chloramphenicol, Streptomycin, Sulfisoxazole, Tetracycline) penta-resistance at $29.06 \%$ (50/172), followed by the MDR-AmpC (ACSSuT phenotype + resistance to Amoxacillin and Ceftiofur) phenotype with a total of $18.02 \%(31 / 172)$. In cattle, the predominant phenotype was ACSSuT making up to 42.99\% (46/107) of the total MDR isolates in cattle followed by resistance to at least MDR-AmpC $21.50 \%$ (23/107). In humans the majority of MDR isolates displayed the MDR-AmpC pattern $-12.31 \%(8 / 65)$ followed by the phenotype resistant to Gentamycin, Streptomycin and Sulfisoxazole $(7.8 \%, 5 / 65)$. Of all the MDR-AmpC isolates observed in both cattle and humans, 5 of them had resistance to Nalidixic acid three of which also had resistance to Trimethoprim; 11 of them were resistant to Trimethoprim only (used for the treatment of invasive salmonellosis). Presence of the MDR phenotype ACSSuT or MDR-AmpC was not significantly associated with presence of integron 1 ( $\mathrm{p}$ value $<0.05$ ). Out of all the multidrug resistant isolates $(\geq 2)$ only $2(1.16 \%)$ were resistant to Nalidixic acid while $54(31.40 \%)$ were resistant to Ceftiofur. From Uganda, out of 73 Salmonella isolates tested $74.6 \%$ (54/73) were resistant to $\geq 5$ antimicrobials while $94.4 \%$ (69/74) were resistant to at least 2 (Figure 3). For cattle and human isolates $100 \%(14 / 14)$ and $93.1 \%$ (55/59), respectively, had resistance to $\geq 2$ antimicrobials. There were no pan susceptible isolates in both cattle and human isolates from Uganda (Figure 3).

\section{Prevalence of Class 1 and 2 integrons}

A total of $20.70 \%(57 / 276)$ of the Salmonella isolates from North Dakota were positive for presence of the integrase 1 gene - indicative of class 1 integron presence. Of these, $26.7 \%(32 / 120)$ were cattle and $16.02 \%(25 / 156)$ were human isolates. Presence of class 1 integron in the Salmonella isolates was significantly associated with antimicrobial

\begin{tabular}{|c|c|c|c|c|c|c|c|c|}
\hline \multirow[b]{2}{*}{ Antimicrobial } & \multicolumn{4}{|c|}{ Humans Isolates } & \multicolumn{4}{|c|}{ Animal isolates (cattle) } \\
\hline & $\begin{array}{c}\text { (\%)North Dakota } \\
{[n=186]}\end{array}$ & $\begin{array}{l}\text { (\%) Uganda } \\
{[n=58]}\end{array}$ & Chi square value & $P$ value & $\begin{array}{c}\text { (\%)North Dakota } \\
{[n=172]}\end{array}$ & $\begin{array}{l}\text { (\%) Uganda } \\
{[n=14]}\end{array}$ & Chi square value & $P$ value \\
\hline Amikacin & 0.0 & 0.0 & & & 0.6 & 16.7 & & \\
\hline Ceftiofur & 4.3 & 28.6 & 30.07 & $<0.0001$ & 28.1 & 41.7 & 0.71 & 0.39923 \\
\hline Amoxcia & 11.3 & 73.7 & 90.25 & $<0.0001$ & 42.8 & 50.0 & 0.28 & 0.59974 \\
\hline Ampicillin & 13.4 & 16.1 & 0.16 & 0.69013 & 53.2 & 23.7 & 5.22 & 0.02228 \\
\hline Cefoxitin & 5.9 & 8.8 & ${ }^{*} 0.18$ & 0.67208 & 29.1 & 25.0 & 0.08 & 0.77922 \\
\hline Ceftriaxone & 3.2 & 14.8 & ${ }^{*} 9.54$ & 0.00201 & 17.3 & 16.7 & 0.01 & 0.93868 \\
\hline Chloramphenicol & 16.7 & 81.0 & 84.23 & $<0.0001$ & 47.4 & 50.0 & 0.04 & 0.85131 \\
\hline Ciprofloxacin & 0.0 & 14.3 & *22.35 & $<0.0001$ & 1.2 & 27.3 & & \\
\hline Gentamycin & 4.8 & 44.2 & 57.54 & $<0.0001$ & 13.8 & 25.0 & 1.2 & 0.27432 \\
\hline Kanamycin & 10.2 & 49.0 & 41.18 & $<0.0001$ & 32.4 & 16.7 & 1.22 & 0.26844 \\
\hline Nalidixic acid & 5.4 & 44.9 & 57.54 & $<0.0001$ & 4.6 & 72.7 & *58.99 & $<0.0001$ \\
\hline Streptomycin & 16.4 & 77.2 & 78.44 & $<0.0001$ & 54.8 & 36.4 & 1.92 & 0.16598 \\
\hline Sulfizoxazole & 16.1 & 92.9 & 30.21 & $<0.0001$ & 51.5 & 83.3 & 6.12 & 0.01334 \\
\hline Tetracycline & 19.4 & 65.4 & 44.59 & $<0.0001$ & 61.3 & 58.3 & 0.09 & 0.76068 \\
\hline Trimethoprimsulp & 1.1 & 85.7 & 191.08 & $<0.0001$ & 7.5 & 63.6 & 34.63 & $<0.0001$ \\
\hline
\end{tabular}

Table 2: A comparison of the proportions in resistance in both human and cattle isolates for panel of antimicrobials.

\begin{tabular}{|c|c|c|c|c|c|}
\hline Antimicrobial & Odds ratio & Lower $\mathrm{Cl}$ & Upper Cl & P-values & Attributable Fraction \\
\hline Amikacin & Undef & Undef & Undef & 0.04 & \\
\hline AMOX/CLA & 1.75 & 0.92 & 3.33 & 0.04 & \\
\hline Ampicillin & 2.78 & 1.50 & 5.14 & $<0.01$ & $33.84 \%$ \\
\hline Cefoxitin & 0.87 & 0.14 & 1.20 & 0.14 & \\
\hline Ceftiofur & 0.90 & 0.39 & 2.08 & 0.41 & \\
\hline Ceftriaxone & 1.08 & 0.41 & 2.78 & 0.43 & \\
\hline Chloramphenicol & 1.02 & 0.53 & 1.96 & 0.47 & \\
\hline Ciprofloxacin & Undef & Undef & Undef & 0.01 & \\
\hline Gentamicin & 2.21 & 0.89 & 5.48 & 0.27 & \\
\hline Kanamycin & 2.56 & 1.31 & 5.01 & $<0.01$ & $17.13 \%$ \\
\hline Nalidixic acid & 1.55 & 0.04 & 4.96 & 0.48 & \\
\hline Streptomycin & 2.36 & 1.34 & 4.94 & 0.02 & $33.07 \%$ \\
\hline Sulfisoxazole & 3.13 & 1.69, & 5.82 & $<0.01$ & $37.26 \%$ \\
\hline Tetracycline & 2.12 & 1.16 & 3.90 & $<0.01$ & $29.92 \%$ \\
\hline Trimethoprim & 1.39 & 0.27 & 7.11 & 0.34 & \\
\hline
\end{tabular}

Table 3: Association of Antimicrobial Resistance and Presence of Class 1 Integron among Salmonella isolates from North Dakota. 
Citation: Mahero M, Byarugaba DK, Doetkott DK, Olet S, Khaitsa ML (2013) Antimicrobial Resistance and Presence of Class 1 Integrons in Salmonella Serovars Isolated from Clinical Cases of Animals and Humans in North Dakota and Uganda. Clin Microbial 2: 128. doi: 10.4172/23275073.1000128

Page 5 of 7

resistance to: Ampicillin (OR 2.78; CI 1.50, 5.14; p-value Fishers exact $<0.001$ ); Kanamycin (OR 2.56; CI 1.31, 5.01; p-value Fishers exact < 0.001); Tetracycline (OR 2.12; CI 1.16, 3.90; p-value Fishers exact 0.02) , Streptomycin (OR 2.58; CI 1.34, 4.94; p-value Fishers exact < 0.02) and Sulfisoxazole (OR 3.132; CI 1.69, 5.82; p-value < 0.001) (Table 2). Of the samples from Uganda, a total of $45.80 \%$ (33/72) tested positive for presence of integrase 1 gene. Of these, $45.80 \%$ (27/59) were human and $46.20 \%(6 / 13)$ were cattle isolates. Out of a subset of 30 isolates from Uganda $3(10 \%)$ of them tested positive for integron 2. There were higher proportions $(47.9 \%, 34 / 72)$ of integron positive MDR Salmonella isolates from Uganda compared to those from ND $(29.85 \%$, 40/134). Presence of class 1 integron was significantly associated with AMR to Tetracycline (OR 5.94, CI 1.85, 19.09; p-value < 0.001) and Amoxicillin (OR 4.41; CI 1.442, 13.497, p-value < 0.01) (Table 3).

\section{Association of class 1 integron to the observed antimicrobial resistance}

Up to $32.35 \%$ (22/68) of MDR isolates ( $>5$ antimicrobials) had the integrase 1 gene. Of these $17(30.90 \%)$ were from cattle and 5 $(38.50 \%)$ from humans. An attributable fraction (AF) and significant associations were computed to quantify role of class 1 integron in MDR Salmonella. Significant $(\mathrm{P}$-value $<0.01)$ AF values for the isolates from

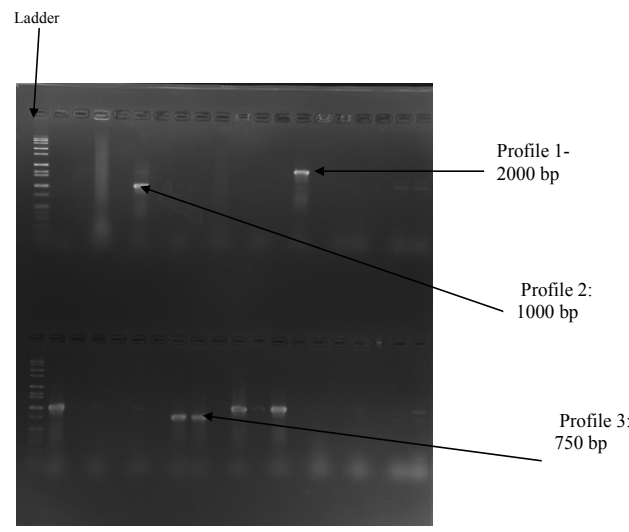

Figure 4: DNA Sequencing Results; $1.5 \%$ Agarose Gel showing different sized Integron 1 Gene Cassette amplicons. Amplification of isolates was done using their conserved sequences (CS). MW: Hi-Lo TM DNA Ladder was used.
ND were recorded against: Ampicillin 33.84\%; Sulfizoxazole $37.26 \%$; Streptomycin $33.07 \%$; Kanamycin $17.13 \%$; Tetracycline $29.92 \%$. Among the isolates from Uganda $36.31 \%$ of resistance towards Amoxicillin and $65.20 \%$ of Tetracycline was attributed to presence of class 1 integron (Table 2).

\section{DNA sequencing}

After amplification the most frequently encountered profile had a 1000 bp followed by a 750 bp amplicon (Figure 4). Only $63.33 \%$ (57/90) of Integrase 1(Int 1) positive Salmonella isolates from North Dakota contained the integron conserved sequence in their integration site. In order to determine the content of the variable regions the detected amplicons were subjected to DNA sequencing. Among these North Dakota isolates 2 gene cassette profiles were detected (1000 bp and $750 \mathrm{bp}$ ). Sequencing of the $1000 \mathrm{bp}$ amplicon identified mainly the aadA family of genes including; aadA1 which confer resistance to Streptomycin and Spectinomycin; additionally acetyltransferase (aac(6')-Ib-cr) which confers resistance to Amikacin, Tobramycin and Kanamycin was also identified; however, the 750 bp mainly contained the dfrAl gene. Among the isolates from Uganda all 3 gene cassette profiles were detected (Figure 4). In one isolate two different profiles were identified. The identified cassettes were aadAl which confer resistance to Streptomycin and Spectinomycin; dihydrofolate reductase dfrA7, dfrA5, dfrA1 which confer resistance to Trimethoprim and aminoglycoside acetyltransferase, (aac(6')-Ib-cr) which confers resistance to Amikacin, Tobramycin and Kanamycin, the most common profile had a combination of more than one of these genes.

\section{Discussion}

This study supports previous reports [19-23] that antimicrobial resistance in Salmonella is both a human and veterinary problem. This is further backed by reports from the USDA [24] which state that approximately $25 \%$ of small feedlot cattle operations and $70 \%$ of large feedlot operations use antimicrobials in their feed. In ND, observed resistance against Tetracycline, Streptomycin, Sulfisoxazole, Ampicillin and Chloramphenicol was in tandem with, although slightly lower than, reports from four other state veterinary diagnostic laboratories in the US [22]. Also, Salmonella isolates from ND showed lower resistance towards Tetracycline, Streptomycin, Sulfisoxazole, Ampicillin and Chloramphenicol compared to isolates from Uganda where greatest resistance was towards Tetracycline, Streptomycin, Sulfisoxazole,

\begin{tabular}{|c|c|c|c|c|c|}
\hline Antimicrobial & Odds ratio & Lower $\mathrm{Cl}$ & Upper Cl & P-Values & Attributable Fraction \\
\hline Amikacin & 4.14 & 0.4893 & 22.58 & 0.2185 & \\
\hline AMOX/CLA & 4.41 & 1.44 & 13.50 & 0.0048 & $36.31 \%$ \\
\hline Ampicillin & 1.766 & 0.62 & 5.03 & 0.013 & \\
\hline Cefoxitin & 1.12 & 0.32 & 22.46 & 0.52 & \\
\hline Ceftiofur & 1.10 & 0.25 & 4.82 & 0.61 & \\
\hline Ceftriaxone & 2.0 & 0.58 & 7.88 & 0.26 & \\
\hline Chloramphenicol & 1.38 & 0.46 & 4.16 & 0.27 & \\
\hline Ciprofloxacin & 0.74 & 0.21 & 2.62 & 0.42 & \\
\hline Gentamicin & 2.10 & 0.69 & 6.03 & 0.09 & \\
\hline Kanamycin & 0.84 & 0.32 & 2.44 & 0.47 & \\
\hline Nalidixic acid & 0.93 & 0.12 & 7.09 & 0.62 & \\
\hline Streptomycin & 2.56 & 0.91 & 8.39 & 0.06 & \\
\hline Sulfisoxazole & 5.50 & 0.61 & 49.80 & 0.11 & \\
\hline Tetracycline & 5.94 & 1.85 & 19.09 & $<0.01$ & 69.23 \\
\hline Trimethoprim & 3.60 & 0.88 & 14.75 & 0.40 & \\
\hline
\end{tabular}

Table 4: Association of Antimicrobial Resistance and Presence of Class 1 Integron among Salmonella isolates from Uganda. 
Citation: Mahero M, Byarugaba DK, Doetkott DK, Olet S, Khaitsa ML (2013) Antimicrobial Resistance and Presence of Class 1 Integrons in Salmonella Serovars Isolated from Clinical Cases of Animals and Humans in North Dakota and Uganda. Clin Microbial 2: 128. doi: 10.4172/23275073.1000128

Ampicillin and Chloramphenicol. This difference could be attributed to the easy access to antimicrobials by the general public in Uganda as compared to the US caused by poor prescription and drug adherence in Uganda [25].

Other studies have reported that in Africa, multidrug-resistant non-typhoidal salmonellae (NTS) are one of the leading causes of morbidity and high mortality in children less than 5 years of age [26]. In a study conducted in Nairobi, Kenya [26] the majority of NTS obtained from cases were Salmonella enterica serotype Typhimurium (106 out of 193; 54.9\%) and Salmonella enterica serotype Enteritidis (64; 33.2\%), a significant proportion $(34.2 \%)$ of which were resistant to three or more antibiotics, including ampicillin, tetracycline, cotrimoxazole and chloramphenicol. In this study [26] $23.4 \%$ of NTS were fully susceptible to all 10 antibiotics that were tested while not a single isolate from Uganda was pan susceptible. Resistance observed in Salmonella isolates from Uganda reflected levels previously recorded among isolates from food animal species in Uganda [25]. Among cattle isolates resistance was high to drugs commonly used in Uganda; Tetracycline, Penicillin, Trimethoprim, Ampicillin, and Chloramphenicol, and less resistant to antibiotics less commonly used in the animal industry in Uganda including Amikacin, Ciprofloxacin, Ceftiofur, Cefoxitin [25]. Similarly this trend was also seen among human isolates from Uganda where low resistance was seen against Ciprofloxacin and Cefoxitin. However, high resistance was recorded against Chloromphenical, Trimethoprim, Gentamicin and Tetracycline as reported before [20] possibly due to management of bacteraemia among clinical cases in Kampala. The high resistance against Gentamicin had not been reported before, and is of concern because Gentamicin is currently recommended in combination with Ampicillin for the management of presumed bacteraemia in severely malnourished children [20].

In the US, third-generation cephalosporins (such as Ceftriaxone) and fluoroquinolones (such as Ciprofloxacin) are choice drugs for the treatment of Salmonella infections in humans [27]. The emergence of isolates resistant to Nalidixic acid with reduced susceptibilities to Ciprofloxacin is of great concern given the possibility of treatment failures [27]. Moreover the AMR patterns observed revealed higher resistance to Nalidixic acid among humans compared to cattle possibly due to flouroquinolone use in the treatment of invasive salmonellosis in adults which might have led to cross resistance [28]. High resistance of Salmonella isolates from cattle in the US to beta -lactam antimicrobials was observed. While this could be attributed to the occurrence of multiple drug resistant isolates, the specific use of some of these drugs in animal medicine, such as Ceftiofur (FDA approved for the treatment of bovine respiratory diseases) [1] could explain this phenomenon. This could also explain the considerable resistance observed against Ceftriaxone a drug in the same class with Ceftiofur (cross resistance) which is not used in animal medicine but is indicated for treatment of invasive salmonellosis in children [27]. Relatively low resistance to these set of drugs, Ceftriaxone, Cefoxitin, Ampicillin, Ciprofloxacin from the isolates from Uganda could be attributed to low access and the high cost of these drugs as previously reported [20,25]. Similarly the higher resistance against Kanamycin in the cattle isolates from ND could be attributed to cross resistance to Neomycin used in cattle for the control of E. coli associated morbidity and mortality. Interestingly, in Uganda resistance levels against Kanamycin in cattle were low which was contrary to what was observed in ND isolates and could possibly be due to low access to the drug in Uganda.

Unlike in humans, Chloramphenicol resistance in cattle was associated with presence of the penta-resistance phenotypeACSSuT (Ampicillin, Chloramphenicol, Streptomycin, Sulfisoxazole and Tetracycline). This could be attributed to the prohibition of Chloramphenicol in food animals by the FDA because of its potential to induce aplastic anaemia in humans [29], whereas its use in human medicine still continues, for infections where other antimicrobials are not effective or contraindicated; sustained use of a drug could result in selection of resistance genes among commensal and pathogenic bacteria. Results from this study indicated a difference in the antimicrobial susceptibility of Salmonellae in different hosts, and from different geographical regions. Cattle isolates displayed a higher resistance than those from humans in North Dakota; the opposite was true for the isolates from Uganda possibly because the ND cattle isolates were from were clinical cases. Resistance patterns observed were similar to those seen among clinical NTS isolates from the region [30]. Selective pressure could also results in the proliferation and dissemination of such drug resistant strains [3,31]. Among the ND isolates the multidrug resistant ACSSuT phenotype was the predominant phenotype as previously reported [29]. This phenotype has been linked to the emergence and spread of the multi drug resistant S. Typhimurium DT-104 [13]. The majority $(75 \%, 21 / 28)$ of the MDR-AmpC isolates from the US were recovered from cattle. This is in agreement with previous reports [32] of its recovery only from diseased cattle. This finding has significant implications both in human and animal medicine [32]. In this study, 11(39\%) of the MDR-AmpC isolates were also resistant to Trimethoprim-Sulfamethoxazole, 3 (11\%) were also resistant to Nalidixic acid while $2(7 \%)$ were resistant to Nalidixic acid only. Resistance against Nalidixic acid is a marker for the emergence of flouroquinolone resistance or reduced susceptibilities. Higher resistance against Ciprofloxacin and Nalidixic acid among the isolates from Uganda, especially the humans could possibly be attributed to the easy access to and questionable handling of these drugs [25].

The high incidence of integrons reported from the Salmonella isolates tested points toward their role in the spread of resistant genes; previous studies $[23,33,30]$ have reported similar prevalence of class 1 integrons in Salmonella. Also, in this study we reported significant associations between resistance to several antimicrobials and presence of class 1 integrons. According to our study, class 1 integron explained a sizeable proportion of the multidrug resistant profiles observed. However not all MDR isolates had presence of integrons. Up to 51.4\% (37/72) and 70\% (251/359) of multi drug resistant Salmonella isolates from Uganda and ND, respectively, did not have class 1 integrons further confirming the presence of other mechanisms that mediate the observed resistance. This was supported by the observed attributable fractions $(\mathrm{AF})$ of $<100 \%$ indicating that other mechanisms that mediate the observed resistance existed. Additionally,the high frequency of dfra1 (Trimethoprim) and aadA1 genes (Streptomycin) was not a surprise due to previous reports in the literature [34]. We observed that for the ND isolates, resistance against Streptomycin and Trimethoprim was largely mediated by presence of class 1 integron as had been earlier reported [9]. It is possible that Class 2 integrons also contributed to the carriage and dissemination of antimicrobial resistance genes in Uganda. Further research could focus on quantifying this association by estimating AFs for other mechanisms that code for AMR in Salmonella isolates.

Among Salmonella isolates from Uganda that were subjected to DNA sequencing, one isolate depicted substantial similarity (91\%) to the Salmonella enterica subsp. enterica serovar Typhimurium plasmid pSLT-BT that was identified in Malawi and Kenya. This isolate was implicated in an epidemic of multiple drug resistant Salmonella Typhimurium causing invasive disease in sub-Saharan Africa [6]. This isolate had several resistant genes including aadA1 and dfrAlgene. Although this study provided useful information on AMR and possible 
Citation: Mahero M, Byarugaba DK, Doetkott DK, Olet S, Khaitsa ML (2013) Antimicrobial Resistance and Presence of Class 1 Integrons in Salmonella Serovars Isolated from Clinical Cases of Animals and Humans in North Dakota and Uganda. Clin Microbial 2: 128. doi: 10.4172/23275073.1000128

Page 7 of 7

mediating mechanisms in Salmonella isolates from Uganda and ND, its widespread application was limited because archived samples were used therefore information on prior use of antimicrobials and previous history of hospitalization, which may be associated with bacterial resistance, was not established. .

\section{Conclusion}

These data indicated high AMR among antimicrobials widely used in veterinary and human medicine with several Salmonella isolates exhibiting multidrug resistance. AMR was observed against drugs whose veterinary use is restricted, implying possible horizontal transmission. To the best of our knowledge this was the second account of the role of integron 1 among Salmonellae in Uganda. These results signal serious implications in the treatment of salmonellosis in both public and animal health and underscore the need for further research into mechanisms that mediate antimicrobial resistance among Salmonellae.

\section{References}

1. Allen KJ, Poppe C (2002) Occurrence and characterization of resistance to extended-spectrum cephalosporins mediated by beta-lactamase CMY-2 in Salmonella isolated from food-producing animals in Canada. Can J Vet Res 66: $137-144$

2. CDC (2006) Food Net Surveillance Report for 2004 (Final Report),

3. Vidaver AK (2002) Uses of antimicrobials in plant agriculture. Clin Infect Dis 34 Suppl 3: S107-110.

4. World Health Organization (2002) Antimicrobial resistance. vol. Fact sheet 194. World Health Organization, Geneva, Switzerland.

5. Khaitsa ML, Kegode RB, Bauer ML, Gibbs PS, Lardy GP, et al. (2007) A longitudinal study of Salmonella shedding and antimicrobial resistance patterns in North Dakota feedlot cattle. J Food Prot 70: 476-481.

6. Okeke I, Aboderin NO, Byarugaba DK, Ojo KK, Opintan JA (2007) Growing problem of multidrug-resistant enteric pathogens in Africa. Emerg Infect Dis 13: $1640-1646$

7. Sunde M, Fossum K, Solberg A, Sørum H (1998) Antibiotic resistance in Escherichia coli of the normal intestinal flora of swine. Microb Drug Resist 4 : 289-299.

8. NARMS (2013) National Antimicrobial Resistance Monitoring System.

9. Mead PS, Slutsker L, Dietz V, McCaig LF, Bresee JS, et al. (1999) Food-related illness and death in the United States. Emerg Infect Dis 5: 607-625.

10. Threlfall EJ, Ward LR, Frost JA, Willshaw GA (2000) Spread of resistance from food animals to man--the UK experience. Acta Vet Scand Suppl 93: 63-68.

11. Scallan E, Angulo FJ, Tauxe RV, Hoekstra RM, Griffin PM, et al. (2011) Foodborne Illness Acquired in the United States-Unspecified Agents. Emerg Infect Dis 17: 16-22.

12. Goldstein C, Lee MD, Sanchez S, Hudson C, Phillips B, et al. (2001) Incidence of class 1 and 2 integrases in clinical and commensal bacteria from livestock, companion animals, and exotics. Antimicrob Agents Chemother 45: 723-726.

13. Gebreyes WA, Thakur S, Morrow WE (2006) Comparison of prevalence, antimicrobial resistance, and occurrence of multidrug-resistant Salmonella in antimicrobial-free and conventional pig production. J Food Prot 69: 743-748.

14. Molla B, Miko A, Pries K, Hildebrandt G, Kleer J, et al (2007) Class 1 integrons and resistance gene cassettes among multidrug resistant Salmonella serovars isolated from slaughter animals and foods of animal origin in Ethiopia. Acta Trop 103: 142-149.

15. Dargatz DA, Strohmeyer RA, Morley PS, Hyatt DR, Salman MD (2005) Characterization of Escherichia coli and Salmonella enterica from cattle feed ingredients. Foodborne Pathog Dis 2: 341-347.

16. Miko A, Pries K, Schroeter A, Helmuth R (2005) Molecular mechanisms of resistance in multidrug-resistant serovars of Salmonella enterica isolated from foods in Germany. J Antimicrob Chemother 56: 1025-1033.

17. Nde CW, Logue CM (2008) Characterization of antimicrobial susceptibility and virulence genes of Salmonella serovars collected at a commercial turkey processing plant. J Appl Microbiol 104: 215-223

18. Khaitsa ML, Oloya J, Doetkott D, Kegode R (2008) Antimicrobial resistance and association with class 1 integrons in Escherichia coli isolated from turkey meat products. J Food Prot 71: 1679-1684.

19. Zhao S, Fedorka-Cray PJ, Friedman S, McDermott PF, Walker RD, et al (2005) Characterization of Salmonella Typhimurium of animal origin obtained from the National Antimicrobial Resistance Monitoring System. Foodborne Pathog Dis 2: $169-181$.

20. Bachou H, Tylleskar T, Kaddu-Mulindwa DH, and. Tumwine JK (2006) Bacteraemia among severely malnourished children infected and uninfected with the human immunodeficiency virus-1 in Kampala, Uganda. BMC Infect Dis 6: 160 .

21. Foley SL, Lynne AM (2008) Food animal-associated Salmonella challenges: pathogenicity and antimicrobial resistance. J Anim Sci 86: E173-187.

22. Yang B, Zheng J, Brown EW, Zhao S, Meng J (2009) Characterisation of antimicrobial resistance-associated integrons and mismatch repair gene mutations in Salmonella serotypes. Int J Antimicrob Agents 33: 120-124.

23. Zhao S, McDermott PF, White DG, Qaiyumi S, Friedman SL, et al. (2007) Characterization of multidrug resistant Salmonella recovered from diseased animals. Vet Microbiol 123: 122-132.

24. APHIS (2007) Antimicrobial Resistance Issues In Animal Agriculture.

25. Byarugaba DK, Kisame R, Olet S (2011) Multi-drug resistance in commensal bacteria of food of animal origin in Uganda. African Journal of Microbiology Research 5: 1539-1548.

26. Kariuki S, Revathi G, Kariuki N, Kiiru J, Mwituria J, et al (2006) Invasive multidrug-resistant non-typhoidal Salmonella infections in Africa: zoonotic or anthroponotic transmission? J Med Microbiol 55: 585-91.

27. Threlfall EJ, Frost JA, Ward LR, Rowe B (1996) Increasing spectrum of resistance in multiresistant Salmonella typhimurium. Lancet 347: 1053-1054.

28. World Health Organization (2002) Use of antimicrobials outside human medicine and resultant antimicrobial resistance in humans.

29. Constable PD (2004) Antimicrobial use in the treatment of calf diarrhea. J Ve Intern Med 18: 8-17.

30. Kariuki S, Gilks C, Corkill J, Kimari J, Benea A, et al. (1996) Multi-drug resistant non-typhi salmonellae in Kenya. J Antimicrob Chemother 38: 425-434.

31. Frank T, Gautier V, Talarmin A, Bercion R, Arlet G (2007) Characterization of sulphonamide resistance genes and class 1 integron gene cassettes in Enterobacteriaceae, Central African Republic (CAR). J Antimicrob Chemothe 59: $742-745$

32. Threlfall EJ (2000) Epidemic salmonella typhimurium DT 104--a truly international multiresistant clone. J Antimicrob Chemother 46: 7-10.

33. Guerra B, Soto S, Helmuth R, and Mendoza MS (2002) Characterization of a self-transferable plasmid from Salmonella enterica serotype typhimurium clinical isolates carrying two integron-borne gene cassettes together with virulence and drug resistance genes. Antimicrob Agents Chemother 46: 2977 2981.

34. Zhao S, White DG, Friedman SL, Glenn A, Blickenstaff K, et al. (2008) Antimicrobial resistance in Salmonella enterica serovar Heidelberg isolates from retail meats, including poultry, from 2002 to 2006. Appl Environ Microbio 74: 6656-6662. 